\title{
Specificity of Antibodies against Neisseria gonorrhoeae That Stimulate Neutrophil Chemotaxis

\author{
Role of Antibodies Directed against Lipooligosaccharides
}

Peter Densen, Sunita Gulati, and Peter A. Rice

Department of Medicine, Veterans Administration Medical Center, and The University of Iowa Hospitals and Clinics, Iowa City, Iowa 52242; and The Maxwell Finland Laboratory for Infectious Diseases, The Department of Medicine, Boston City Hospital,

Boston University School of Medicine, Boston, Massachusetts 02118

\section{Abstract}

Five strains each of Neisseria gonorrhoeae sensitive or resistant to complement $(C)$ dependent killing by normal human serum (NHS) were examined for their ability to stimulate chemotaxis of polymorphonuclear leukocytes (PMNs) after preincubation with NHS; or IgM or IgG derived from NHS. Serum-sensitive $N$. gonorrhoeae stimulated C-dependent chemotaxis when opsonized with IgM, but not IgG, however, serum-resistant strains, taken as a whole, failed to promote chemotaxis when opsonized with either isotype.

IgM titers in NHS against lipooligosaccharide (LOS) antigens from individual serum-sensitive, but not serum-resistant strains, correlated with the magnitude of chemotaxis generated by the corresponding opsonized strains $(r=0.99)$. Western blots demonstrated that IgM and IgG from NHS recognized different antigenic determinants on LOS from serum-sensitive gonococci. IgM from NHS immunopurified against serum-sensitive LOS accounted for two-thirds of the chemotaxis promoting activity present in whole serum.

IgG titers in NHS against LOS antigens from individual serum-resistant strains also correlated with magnitude of chemotaxis generated by the corresponding opsonized strains ( $r$ $=0.87$ ), although most opsonized serum-resistant strains did not generate significantly higher magnitudes of chemotaxis than controls. In contrast, a serum-resistant isolate from a patient with disseminated gonococcal infection (DGI) stimulated chemotaxis when opsonized with IgG obtained from the patient's convalescent serum. By Western blot, convalescent IgG antibody recognized an additional determinant on serum-resistant LOS not seen by normal IgG.

\section{Introduction}

Neisseria gonorrhoeae are capable of causing a diverse array of syndromes in the hosts they infect. These syndromes range from the total absence of signs or symptoms at the local site of infection, as occurs commonly in disseminated gonococcal infection (DGI), ${ }^{1}$ to a marked local inflammatory response as exemplified

Received for publication 22 August 1986 and in revised form 28 January 1987.

1. Abbreviations used in this paper: AGS, agammaglobulinemic serum; CFU, colony-forming units; CTX, chemotaxis; DGI, disseminated gonococcal infection; ELISA, enzyme linked immunosorbent assay; GVB,

J. Clin. Invest.

(C) The American Society for Clinical Investigation, Inc.

0021-9738/87/07/0078/10 \$2.00

Volume 80, July 1987, 78-87 by acute salpingitis. A number of attributes have been associated with gonococci that manifest different clinical potential. The most solidly established that bears on the inflammatory potential of the organism at the local site of infection, is the resistance of gonococci causing disseminated infection to the complement (C)-dependent bactericidal action of normal human serum (serum resistance) (1-4). This mechanism may confer upon the organism the ability to escape local defenses, enable it to penetrate mucosal barriers to reach the bloodstream, and then to disseminate. Serum-resistant organisms often persist at local sites of infection without promoting clinically significant inflammation as evidenced by a disproportionate representation of these strains in asymptomatic men (5) and by the frequent lack of local symptoms that accompany disseminated infection $(6,7)$. In contrast, serum-sensitive $N$. gonorrhoeae often are associated with symptomatic local infection in both men and women and may be especially related to the severity of the inflammatory response in women with acute salpingitis (2). Serum-sensitive gonococcal isolates more rapidly generate $\mathrm{C5a}$, the complementderived neutrophil chemoattractant when incubated in normal serum (3) and are more rapidly and completely phagocytosed by neutrophils (PMNs) in vitro (8) than serum-resistant strains. Thus, generation of an inflammatory response by gonococci appears to be conducive to the localization of disease and the prevention of bacteremic spread of infection, as evidenced clinically by the rarity with which acute salpingitis leads to DGI $(6,9,10)$.

Killing of serum-sensitive gonococci in normal human serum (NHS) requires $\operatorname{IgM}(2,11)$ specific for antigenic constituents of gonococcal lipooligosaccharides (LOS) (12). Even at low concentrations these antibodies promote killing, in the presence of small amounts of complement such as might be found in normal cervical mucus (13). We report here the immunoglobulin isotype specificity and the role of antibodies directed against LOS antigens in promoting the generation of complement dependent neutrophil chemotaxis by serum-sensitive and resistant gonococci.

\section{Methods}

Source of immunoglobulins and specific antibodies. Serum was obtained, as described (2), from 20 normal volunteers (10 women and 10 men) who had no history of Neisseria infection and who were taking no medications. Pooled sera from these individuals was used for the fractionation of normal immunoglobulins. A serum from a single donor was used to prepare IgM antibodies specific for LOS antigens from strain 24-1, a

gelatin veronal-buffered saline; KDO, 3-deoxy-manno-octulosonic acid; LOS, lipooligosaccharides; NHS, normal human serum; OMP, outer membrane protein; PAGE, polyacrylamide gel electrophoresis; $P$, piliated; PID, pelvic inflammatory disease; PMN, neutrophils; TEA, triethylamine; $\mathrm{Tr}$, transparent. 
serum-sensitive gonococcal strain. Sera from a patient convalescing from DGI taken at 19, 23, 36 and $61 \mathrm{~d}$ after the onset of symptoms were each examined for the presence of antibodies to major classes of outer membrane antigens prepared from the infecting strain (serum-resistant strain 71-H). The 23-day serum (14), was also fractionated and used in bactericidal and chemotaxis assays that employed the resistant organism that had infected the DGI patient.

Strains. 10 strains of $N$. gonorrhoeae identified by standard methods were used. All were clinical isolates and were chosen because they exhibited resistance or sensitivity to normal human serum as defined previously for these strains (2) and reestablished in these studies. Resistant strains were isolated from three patients with DGI, one with pelvic inflammatory disease (PID) and one patient with uncomplicated genital infection. Sensitive strains were isolated from four patients with PID and one uncomplicated genital infection. Isogenic nonpiliated $\left(\mathrm{P}^{-}\right)$ transparent $(\mathrm{Tr})$ gonococci were used for preparation of antigens and in the chemotaxis studies.

Media and growth conditions. Transparent, nonpiliated phenotypes of gonococci were grown in mass culture on petri dishes containing a clear media of trypticase and thiotone peptones (BBL, Div. Becton, Dickinson \& Co., Cockeysville, MD), plus salts and soluble starch, prepared according to the methods of James and Swanson (15).

Preparation of antigens. LOSs were prepared from whole bacteria using a modification (16) of hot phenol water extraction (17). LOS preparations were judged pure according to the following criteria: (a) elution as a single peak in the void volume of Sepharose 4B (Pharmacia Fine Chemicals, Piscataway, NJ) in gel filtration; (b) $83.5 \%$ of dry weight (mean dry weight of LOSs from the 10 strains) accounted for as: hexose (47.8\% [18]), 3-deoxy-manno-octulosonic acid (KDO) $(7.74 \%[19,20])$, fatty acid (26.85\% [16]) and < 1.1\% protein (21); (c) lack of absorption maxima in the 210 to $300 \mathrm{~nm}$ region of the ultraviolet spectrum (22); and $(d)$ lack of characteristic proteins found in gonococcal outer membranes assessed by sodium-dodecyl sulfate (SDS)-polyacrylamide gel electrophoresis (PAGE) $(23,24)$.

Outer membrane proteins (OMPs) freed of LOS were prepared from outer membranes of each gonococcal strain by detergent chromatography according to methods described previously (14). Less than $0.25 \%$ LOS contamination of OMPs was demonstrated by SDS-PAGE (25) employing a modified (16) silver stain $(26,27)$.

Preparation of antibodies. Immunoglobulins $\mathrm{M}$ and $\mathrm{G}$ were separated from individual and pooled normal human serum, and DGI patient 71 $\mathrm{H}$ immune convalescent serum by sequential molecular sieve and anion exchange chromatography as described previously $(2,14)$.

Antibodies directed against LOS antigens from a single serum-sensitive strain (24-1) were immunopurified from the IgM fraction of normal human serum. LOS from this sensitive strain was used to construct an immunoabsorbant for the purification of anti-LOS antibody. LOS was first modified by hydrazinolysis to generate free amines on the antigen (28) to facilitate coupling by cyanogen bromide activated Sepharose 4B (Pharmacia Fine Chemicals). The hydrazide derivative was characterized in the following manner: (a) the carbohydrate content of the derivative was 1.8 -fold greater than the whole $\operatorname{LOS}(18) ;(b)$ the fatty acid content was $<6 \%(16) ;(c)$ after treatment with $p$-dimethylamino benzaldehyde (29) the derivative released $1 \mu \mathrm{mol}$ of hydrazine as the hydrazide per 3$7.5 \mu \mathrm{mol} \mathrm{LOS}$-hydrazide (assuming a possible MW of LOS-[hydrazide] ranging from 1,800 to $4,500[30,31])$; and $(d)$ the comparative antigenicity $(w t / w t)$ in solid-phase enzyme-linked immunosorbent assay (ELISA) inhibition (32) against the LOS from which it was prepared was $91 \%$. LOShydrazide was coupled to Sepharose 4B (Pharmacia Fine Chemicals) that was activated with cyanogen bromide and triethylamine (TEA) (pH 7.0). This activation process favors the formation of cyanate esters, which are predominantly responsible for covalent coupling of ligand to the resin $(33,34)$. LOS-hydrazide $(30 \mathrm{mg})$ was dissolved in $15 \mathrm{ml}$ of $0.1 \mathrm{M}$ $\mathrm{NaHCO}_{3}$ buffer containing $0.5 \mathrm{M} \mathrm{NaCl}$ (pH 8.3) and gently mixed with the activated gel resuspended in $15 \mathrm{ml}$ of the $\mathrm{NaHCO}_{3}$ buffer solution. After overnight incubation at $4^{\circ} \mathrm{C}$, the remaining reactive groups were blocked by incubating the gel with $0.1 \mathrm{M}$ Tris- $\mathrm{HCl}(\mathrm{pH} \mathrm{8.0)}$ for $2 \mathrm{~h}$ at room temperature.' Control gels were also prepared using mock activation (gonococcal LOS omitted in the coupling reaction), followed by blocking of active sites with Tris- $\mathrm{HCl}$. The IgM preparation $(0.5$ to $1.5 \mathrm{ml})$ was equilibrated in PBS with $0.5 \%$ added Tween-20 and applied to columns containing the gels $\left(4^{\circ} \mathrm{C}\right)$ overnight and the effluents washed through with PBS-Tween. Effluents were absorbed with beads made of a copolymer of styrene-divinylbenzene (Bio-Beads SM-Z, Bio-Rad Laboratories, Richmond, CA) (35) to remove Tween. Additional samples of IgM were applied to columns and the same procedure repeated for a total of three runs through the columns. After a final wash with PBS the columns were brought to room temperature, the PBS exchanged for $2 \mathrm{M} \mathrm{KI}$ and after an hour the antibody was eluted from the columns with additional $2 \mathrm{M}$ $\mathrm{KI}$. Effluents and eluents were exchanged into $0.15 \mathrm{M} \mathrm{NaCl}$, concentrated, and used promptly in the immunologic assays.

ELISA. Direct binding activity for outer membrane antigens was determined by ELISA (36). As described previously (16), several dilutions of each specimen were tested and titers were expressed as the limiting dilution that gave $0.3 \mathrm{OD}$. Coating concentrations of $60 \mu \mathrm{g} / \mathrm{ml}$ for LOS and $10 \mu \mathrm{g} / \mathrm{ml}$ for OMPs were predetermined to give optimal readings. Conjugates were validated for their specificity in recognizing the designated isotype (16). IgM and IgG antibody titers directed against outer membrane antigens were measured with the purified immunoglobulin preparations and in whole sera (convalescent immune and normal) and the activity found to be similar as judged by the endpoint ELISA titers (range: from $-13 \%$ to $+12 \%$ ). These results indicated that isotype specific titers against LOSs measured in whole serum were not reduced by the presence of antibodies of other classes.

Each ELISA experiment was performed on three separate occasions and the standard error of the geometric mean titer of the three results was used as a measure of variance. Differences in mean titers of isotype specific ELISA directed against LOSs from each of the two groups (serum sensitive and resistant) were compared using the two-tailed $t$ statistic.

Western blots. The specificity of IgM and IgG for LOS antigens was examined further in Western blotting experiments (37). LOSs from two strains were chosen for study; the serum-sensitive LOS from strain 24 1 used for immunopurification of normal IgM and the resistant LOS isolated from the DGI strain 71-H used in studies of convalescent immune serum from the patient infected with this strain. LOS antigens $(8 \mu \mathrm{g} /$ lane) were first separated by SDS-PAGE $(23,24)(14 \%$ acrylamide) and then electroblotted to Immobilon PVDF Transfer Membrane (Millipore Corp., Bedford, MA) in a solution of $20 \%$ methanol (vol/vol) containing $0.025 \mathrm{M}$ Tris and $0.192 \mathrm{M}$ glycine (pH 8.3). LOS transfer was carried out at constant current $(250 \mathrm{~mA})$ for $2 \mathrm{~h}$ at room temperature and then washed in PBS with $10 \%$ added natural non-fat dry milk (Carnation Co., Los Angeles, CA). Antibody containing solutions were diluted in 1\% bovine serum albumin (BSA)-PBS with $0.5 \mathrm{M} \mathrm{NaCl}$ and shaken together with strips of the transfer membrane containing the desired LOS antigen for $16 \mathrm{~h}$ at $4^{\circ} \mathrm{C}$. Specificity of binding was identified with alkaline phosphatase conjugated goat anti-IgM or anti-IgG (Sigma Chemical Co.) and visualized after the addition of substrate containing nitrobluetetrazolium and indoxyl phosphate in dimethylformamide (38).

Bactericidal assay. The bactericidal assay used in this study was a modification of a previously described procedure (39). Sera, immunoglobulin preparations or antibodies prepared by affinity chromatography were used as sources of antibodies in the bactericidal reactions and were diluted with Gey's balanced salt solution. The complement (C) source was the serum of a 27-yr-old woman with acquired agammaglobulinemia $(3,40)$. The concentration of $C$ used with serum-sensitive gonococci was optimized in preliminary experiments that employed excess bactericidal antibody $\left(56^{\circ} \mathrm{C} \times 30 \mathrm{~min}\right)$ and varying concentrations of $\mathrm{C}$. The concentration of $\mathrm{C}$ chosen was twice the lowest concentration that supported maximal killing. The 71-H DGI convalescent immune serum used in this study, when employed in concentrations varying from $0.5 \%$ to $6 \%$, killed the homologous infecting strain (14), and was used at concentrations that coincided with those of IgG and IgM isolated from this serum that were used in the chemotaxis experiments.

Results of bactericidal experiments were expressed as the percent reduction of colony-forming units (CFUs) after the 30-min incubation period, compared with the numbers of organisms present in the same 
tube after immediate plating $\left(t_{0}\right)$. The mean reduction \pm range was determined by performing each assay on two separate occasions. In some experiments titers were assigned to the dilution of serum, immunoglobulin fraction or antibody preparation that produced $>50 \%$ reduction of CFUs.

Chemotaxis experiments. Broth grown log phase gonococci $\left(5 \times 10^{7}\right.$ CFUs/ml of reaction mixture) were incubated at $22^{\circ} \mathrm{C}$ in gelatin veronalbuffered saline (GVB) containing added immunoglobulins from normal serum, DGI convalescent serum or antibodies prepared by affinity chromatography. The resultant mixtures were tumbled for $15 \mathrm{~min}$. These preincubated organisms were retrieved by centrifugation, resuspended in $6 \%$ agammaglobulinemic serum (AGS) in GVB as a source of complement $(\mathrm{C})$ and tumbled slowly at $37^{\circ} \mathrm{C}$ for $10 \mathrm{~min}$. After incubation, the samples were removed, heated for an additional $30 \mathrm{~min}$ at $56^{\circ} \mathrm{C}$ in a static water bath to destroy residual $\mathrm{C}$ activity, and diluted with an equal volume of GVB to a final C concentration of $3 \%$. The samples were stored at $-70^{\circ} \mathrm{C}$, and used as the stimulus in chemotaxis experiments the next day. Organisms or immunoglobulins incubated in $6 \%$ AGS alone served as controls.

Chemotaxis was evaluated by a previously described modification of the Boyden chamber technique $(3,41)$ using ${ }^{51} \mathrm{Cr}$-labeled polymorphonuclear leukocytes (PMNs) and two micropore filters (3- $\mu \mathrm{m}$ pore size, Sartorius Membranfilter, Gottingen, West Germany). The upper compartment of the chemotaxis chamber (Ahlco Scientific Corp., Southington, CT) contained ${ }^{51} \mathrm{Cr}$ labeled human PMNs prepared as described previously (3). The lower chamber compartment contained the chemotactic stimulus. After incubation of chambers at $37^{\circ} \mathrm{C}$ with $5 \%$ carbon dioxide in air and $100 \%$ humidity for $3 \mathrm{~h}$, the amount of radioactivity incorporated into the lower filter was determined. Chemotactic activity was expressed as corrected counts per minute (41). Within a given experiment, each condition was examined in triplicate or quadruplicate chambers; the results were averaged to obtain a single value, and the value of the corresponding control containing $6 \%$ AGS without gonococci, or $6 \%$ AGS, also without gonococci, but supplemented with immunoglobulin(s), was subtracted from the experimental value. Each chemotaxis experiment was performed on three separate occasions and the standard error of the mean of the three results was used as a measure of variance. Using the two-tailed $t$ statistic, the means of experiments that employed different sources of immunoglobulins were compared to those in which organisms were incubated in $6 \%$ AGS in the absence of added immunoglobulins.

\section{Results}

Bactericidal activity of sera and derived immunoglobulins. The bactericidal capacity of sera and their derived immunoglobulin fractions and the relative purity of the immunoglobulin preparations are shown in Table I. Pooled NHS diluted to $3 \%$ in the reaction volume killed $>50 \%$ of the colonies in bactericidal reactions performed with each of the five serum-sensitive strains. The mean and range of killing of these strains is also shown in Table I. Serum-resistant strains were not killed by $50 \%$ NHS. The isolated IgM containing a concentration $4 \%$ of that in NHS produced $>50 \%$ killing of each of the serum-sensitive strains (mean $87 \pm 12 \%$ for all five strains, Table I). IgM did not kill strains that resisted killing by $50 \%$ NHS when used at concentrations greater than those employed in the whole serum reactions ( 90 vs. $62.5 \mathrm{mg} / \mathrm{dl}$, Table I). IgG isolated from NHS was unable to kill strains sensitive or resistant to NHS when tested in reaction mixtures at concentrations similar to those in $50 \%$ NHS (430 vs. $480 \mathrm{mg} / \mathrm{dl}$, Table I). 71-H DGI immune convalescent serum used in concentrations as low as $0.5 \%$ (with complement added separately) killed the homologous infecting strain that resisted killing by NHS. In experiments reported earlier (14), this immune serum, when used at concentrations of $0.26 \%$ or $0.52 \%$, demonstrated bactericidal activity that was eliminated
Table I. Bactericidal Activity* of Human Serum and Derived Immunoglobulin Fractions against $N$. gonorrhoeae Strains

\begin{tabular}{|c|c|c|c|c|}
\hline \multirow[b]{2}{*}{ Immunoglobulin source ${ }^{\ddagger}$} & \multicolumn{2}{|l|}{ \% Kill } & \multirow[b]{2}{*}{$\lg G$} & \multirow[b]{2}{*}{ IgM } \\
\hline & $\operatorname{ser}^{4}(n=5)$ & $\operatorname{ser}^{n}(n=5)$ & & \\
\hline & & & $m g / d l^{\prime \prime}$ & $m g / d l^{\sharp}$ \\
\hline \multicolumn{5}{|l|}{$\begin{array}{l}\text { Normal pooled human } \\
\text { serum }\end{array}$} \\
\hline$(3 \%)$ & $91 \pm 8$ & - & 28.8 & 3.75 \\
\hline$(50 \%)$ & - & $4 \pm 2$ & 480 & 62.5 \\
\hline \multicolumn{5}{|l|}{ IgM fraction' } \\
\hline$(4 \%)$ & $87 \pm 12$ & - & 0.43 & 5.4 \\
\hline$(72 \%)$ & - & $4 \pm 3$ & 7.2 & 90 \\
\hline \multicolumn{5}{|l|}{ IgG fraction** } \\
\hline$(45 \%)$ & $7 \pm 5$ & $4 \pm 3$ & 430 & $<1.5$ \\
\hline \multicolumn{4}{|l|}{ 71-H DGI convalescent } & \\
\hline$(0.5 \%)$ & & $>99 \%$ & 7.44 & 0.4 \\
\hline \multicolumn{5}{|l|}{ IgM fraction } \\
\hline$(66 \%)$ & & $4 \pm 3$ & $<1.16$ & 52.8 \\
\hline \multicolumn{5}{|l|}{ IgG fraction } \\
\hline$(0.5 \%)$ & & $>99 \%$ & 7.02 & $<0.02$ \\
\hline
\end{tabular}

* Bactericidal activity expressed as the mean \pm range of the \% kill for each group of strains. Each strain was tested in two separate assays. ${ }^{\ddagger}$ Nos. in parentheses indicate the concentration in the bactericidal reaction mixtures of designated fraction (whole serum) or the concentration expressed as percent in whole serum (Ig fractions).

ser $^{\text {s }}$, serum-sensitive strains; ser $^{\mathrm{r}}$, serum-resistant strains.

"Ig concentrations in the bactericidal reaction mixtures.

"IgM fraction (prepared by gel filtration over Bio-Gel A-5M [34]).

** IgG fraction (prepared by anion exchange over QAE-Sephadex A50 [36]).

\$ The strain isolated from the patient (DGI) who provided the convalescent serum (71-H convalescent serum) was used to test this serum and its derived Ig fractions.

${ }^{\$ S}$ Strain 71-H (Table II).

by prior absorption with LOS antigen prepared from the infecting strain. In those studies, no loss of bactericidal activity was demonstrated when the serum was absorbed with a mixture of OMPs, freed of LOS, also prepared from the infecting strain (14). These results demonstrated that bactericidal activity in this DGI serum when used at the concentrations indicated in the bactericidal and chemotaxis experiments (see below) was directed principally against LOS. IgG purified from 71-H convalescent serum and employed in similar concentrations also killed this strain. In contrast, IgM purified from immune serum showed no killing of this strain (Table I) even when used in concentrations that exceeded by 138 -fold $(52.8 \mathrm{vs.} 0.4 \mathrm{mg} / \mathrm{dl})$ the concentration of IgM present in $0.5 \%$ convalescent serum.

Chemotactic activity of serum fractions. Gonococci were first incubated with IgM or IgG separated from NHS at immunoglobulin concentrations of 3\%. This was followed by incubation with AGS as a source of complement, to generate the attractant used in the chemotaxis experiments. We have shown previously that in this assay chemotaxis is complement dependent since gonococci incubated in buffer alone did not promote significant activity. In addition the chemotactic factor was identified as C5a by co-chromatography with a standard and by the ability of 
antibody to $\mathrm{C5}$, but not $\mathrm{C3}$, to block chemotactic activity (3). The concentrations of IgG and IgM used in the present experiments (3\% that in whole serum) and complement (6\% AGS) were chosen to simulate reported values for these substances in whole normal cervical mucus $(13,42,43)$. The results of these experiments, illustrated in Fig. 1, portray chemotactic activity that developed during a 30 -min incubation of the two groups of strains with immunoglobulins and AGS. Serum-sensitive gonococci, when incubated with IgM and AGS, generated a significantly higher chemotactic stimulus than control incubations. IgG and then AGS incubated with the serum-sensitive organisms did not enhance chemotaxis (Fig. 1). IgG and IgM combined, and incubated with serum-sensitive gonococci followed by incubation with AGS also resulted in enhanced activity, suggesting primacy of IgM over IgG for chemotaxis by opsonized serumsensitive organisms in serum containing both these immunoglobulins. Taken as a group, incubation of serum-resistant gonococci in NHS, or with immunoglobulin fractions used singly (IgG or IgM) or together (IgG + IgM) failed to promote chemotactic stimulation above that seen in control incubation with AGS alone. However, IgG with high titered antibody against LOSs from two individual serum-resistant strains, promoted significant chemotaxis (see below).

Immunoglobulins from 71-H DGI immune convalescent serum were used in concentrations varying from $0.5 \%$ to $6 \%$ that in whole serum in the preincubations with the infecting resistant strain, followed by incubation with $C$ (Fig. 2). Convalescent $\mathrm{IgG}$, with bactericidal activity directed principally against LOS (14), generated chemotaxis when tested at concentrations as low as $\mathbf{0 . 5 \%}$. The latter concentration was sixfold less than used for testing IgG from NHS. In contrast, IgM purified from the convalescent serum was unable to generate a chemotactic stimulus when incubated with AGS plus the serum-resistant strain.

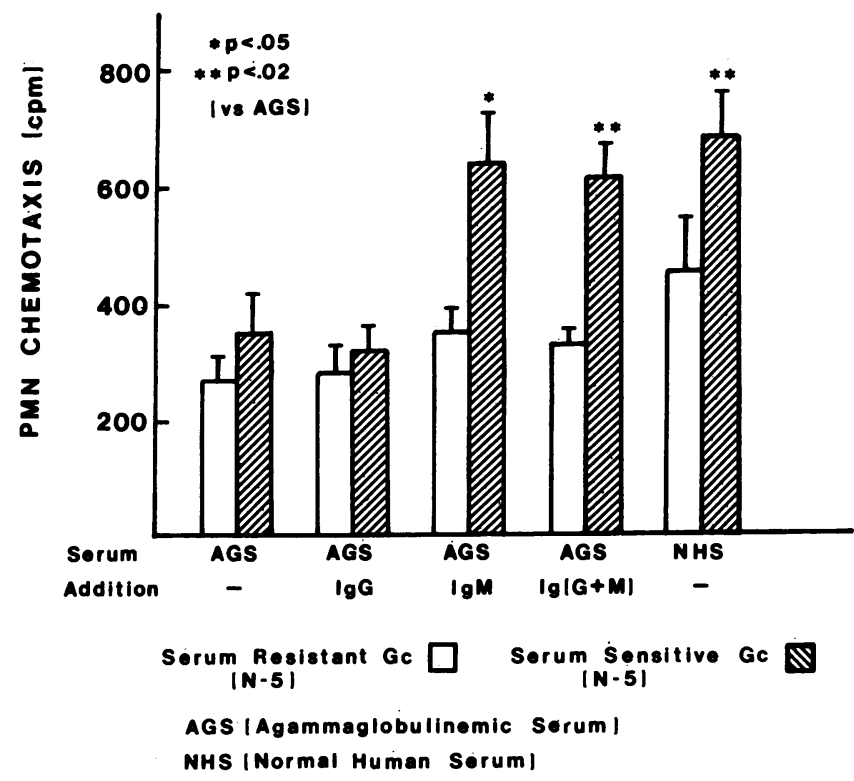

Figure 1. Stimulation of PMN chemotaxis by normal immunoglobulins and gonococci. Gonococci were preincubated $\left(4^{\circ} \mathrm{C}, 30 \mathrm{~min}\right)$ with purified immunoglobulins (at concentrations equivalent to $3 \%$ NHS), then centrifuged organisms were incubated in $6 \%$ AGS $\left(37^{\circ} \mathrm{C}, 30\right.$ $\mathrm{min})$. Results are expressed as mean $\pm \mathrm{SE}$ of experiments performed on three separate occasions. ${ }^{*} P<0.05,{ }^{* *} P<0.02$ (both vs. AGS).

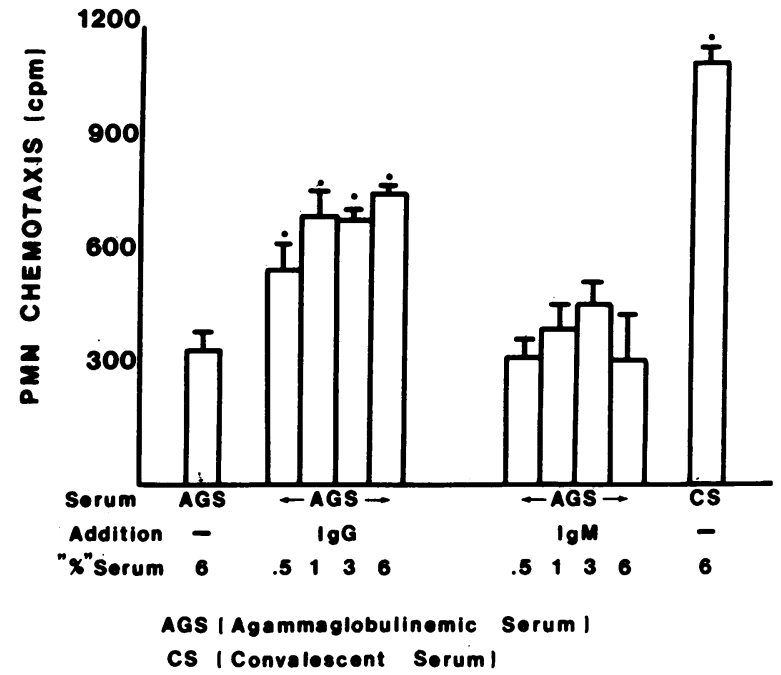

Figure 2. Stimulation of PMN chemotaxis by convalescent DGI immunoglobulins and the homologous infecting serum-resistant gonococcus. Gonococci were preincubated $\left(4^{\circ} \mathrm{C}, 30 \mathrm{~min}\right)$ with immune serum derived immunoglobulin fractions at the concentrations indicated, then centrifuged organisms were incubated in $6 \%$ AGS $\left(37^{\circ} \mathrm{C}\right.$, $30 \mathrm{~min})$. Results are expressed as mean $\pm \mathrm{SE}$ of experiments performed on three sêparate occasions. ${ }^{*} P<0.05$ (vs. AGS).

Antibodies directed against LOS antigens. Additional characterization of chemotaxis mediated by immunoglobulin $\mathbf{M}$ or $\mathrm{G}$ coated serum-sensitive and resistant $N$. gonorrhoeae was performed by measuring titers of isotype specific antibodies in normal and immune sera directed against outer membrane antigens. Isotype specific ELISA activity was measured in the NHS against whole LOS antigens and OMPs prepared from each of the 10 strains (Table II). The development of antibodies directed against these outer membrane antigens prepared from DGI strain 71$\mathbf{H}$ was also measured in sera obtained in convalescence from the patient infected with this strain (Table III). All antibody titers were maximal at $19 \mathrm{~d}$ with levels dropping thereafter. At $61 \mathrm{~d}$ all titers were still higher than those measured in the pool of 20 normal human sera against membrane antigens from the 71-H strain (Table II).

Chemotaxis generated by serum-sensitive strains coated with IgM prepared from NHS showed a linear correlation with IgM titers against corresponding whole LOSs ( $r=0.99$, Table II). IgM titers against corresponding outer membrane protein antigens (OMPs) showed no correlation with chemotaxis mediated by IgM coated serum-sensitive $(r=0.21)$ or serum-resistant ( $r$ $=-0.40$ ) strains. In addition, no significant difference between the geometric mean IgM titer against serum-sensitive vs. resistant OMPs was observed. The mean IgM titer in pooled NHS directed against serum-sensitive LOSs was significantly higher than for serum-resistant LOSs $(2,851 \pm 417$ vs. $1,395 \pm 230$, respectively, $P<0.02$ ), shown in Table IV which summarize NHS antibody data, bactericidal activity and chemotaxis for serum-sensitive and resistant strains and DGI convalescent serum data for strain 71-H. To assess whether inability to mediate chemotaxis by serum-resistant strains was due exclusively to lower anti-LOS titers, we identified a single normal serum with a relatively high titer of anti-LOS antibody against a serum-resistant strain, and measured chemotactic potential of this strain opsonized with the whole serum or the IgM from this serum. This serum (as 
Table II. CTX* Generated by Each of 10 Strains of N. gonorrhoeae Isolates Incubated in IgM or IgG Prepared from Normal Pooled Human Sera and the Corresponding Titers (Reciprocal-ELISA)* Directed against Outer Membrane Antigens Prepared from Each Strain

\begin{tabular}{|c|c|c|c|c|c|c|}
\hline \multirow{2}{*}{$\begin{array}{l}\text { Strain } \\
\text { designation }\end{array}$} & \multicolumn{3}{|l|}{ IgM } & \multicolumn{3}{|l|}{ IgG } \\
\hline & CT: $\mathbf{X}^{*}$ & $\operatorname{LOS}^{ \pm / 1}$ & OMPs" & CTX' & $\operatorname{LOS}^{1 / 3}$ & OMPs" \\
\hline \multicolumn{7}{|c|}{ Serum sensitive } \\
\hline 336 & $987 \pm 187^{* *}$ & $4,365 \pm 513$ & $914 \pm 71$ & $413 \pm 43$ & $5,754 \pm 611$ & $3,212 \pm 99$ \\
\hline $24-1$ & $656 \pm 73^{* *}$ & $3,020 \pm 219$ & $433 \pm 52$ & $267 \pm 26$ & $3,548 \pm 201$ & $1,782 \pm 101$ \\
\hline $\mathrm{i}$ & $558 \pm 90^{* *}$ & $2,636 \pm 119$ & $367 \pm 19$ & $202 \pm 33$ & $3,162 \pm 311$ & $4,320 \pm 217$ \\
\hline 334 & $547 \pm 80^{* *}$ & $2,239 \pm 187$ & $1,315 \pm 101$ & $278 \pm 29$ & $6,818 \pm 206$ & $5,699 \pm 609$ \\
\hline 6863 & $495 \pm 20^{* *}$ & $1,995 \pm 176$ & $515 \pm 60$ & $448 \pm 20$ & $4,074 \pm 512$ & $2,748 \pm 213$ \\
\hline
\end{tabular}

Serum resistant

$\begin{array}{lllllll}\text { RF } & 473 \pm 32^{* *} & 661 \pm 41 & 640 \pm 50 & 436 \pm 105^{* *} & 18,621 \pm 1061 & 5,404 \pm 406 \\ 305 & 318 \pm 80 & 1,950 \pm 98 & 358 \pm 31 & 367 \pm 64^{* *} & 10,471 \pm 898 \\ 7044 & 332 \pm 217 & 1,318 \pm 107 & 436 \pm 97 & 323 \pm 83 & 8,760 \pm 714 & 1,321 \pm 91 \\ 71-H & 208 \pm 37 & 1,820 \pm 231 & 804 \pm 106 & 159 \pm 64 & 7,079 \pm 622 \\ \text { WG } & 423 \pm 15 & 1,230 \pm 106 & 312 \pm 21 & 138 \pm 56 & 5,986 \pm 331 \\ \end{array}$

* Results are expressed as mean (CTX) or geometric mean titers (reciprocal ELISA) of three determinations for each assay \pm SE. ${ }^{\ddagger}$ Linear correlation $(r=0.99)$ between individual values of CTX and individual IgM titers directed against serum-sensitive LOSs. ${ }^{8}$ LOS antigen used as target for ELISA. "OMPs, used as target for ELISA. 'Linear correlation $(r=0.87)$ between individual values of CTX and individual IgG titers directed against serum-resistant LOSs. ${ }^{* *} \mathrm{CTX}>2 \mathrm{SE}$ above control that used complement (6\% agammaglobulinemic serum), but no immunoglobulins.

well as its derived IgM fraction), with an IgM titer (4,510 1311$)$ directed against the LOS of the serum-resistant strain 1.65 times greater than the mean IgM titer of pooled NHS against serumsensitive LOSs, failed to promote chemotaxis when opsonized to this strain. In addition, the IgM fraction prepared from the 71-H DGI convalescent serum with an anti-LOS titer $(3,823 \pm 210)$ for the infecting serum-resistant strain 1.34 times greater than the mean IgM titer of NHS against serum-sensitive LOSs, also failed to promote chemotaxis when opsonized to the 71-H strain even when used at twice the concentration (6 vs. $3 \%$ ) of NHS. In this patient, the IgM antibody titer at $23 \mathrm{~d}$ against LOS was more than twice the titer measured in NHS. Thus, the inability of IgM in normal or convalescent serum to promote chemotaxis by serum-resistant strains is not due solely to lower titers of IgM antibodies, measured by ELISA to whole LOS extracts of resistant strains, or to failure to develop immune IgM antibodies in convalescence.

In order to further assess the role of LOS antibody in the generation of chemotaxis by serum-sensitive gonococci, we immunopurified IgM from normal serum directed against the LOS derived from a serum-sensitive strain (24-1) and examined this antibody in bactericidal and chemotaxis experiments (Table V). The eluent recovered from the LOS-hydrazide-affinity column was concentrated until the bactericidal titer was equivalent to that in NHS and IgM prepared from NHS (1:84). The resultant antibody titer (IgM-ELISA) directed against the LOS used to prepare the column was $3,345 \pm 601$. The concentrated eluent lacked antibody reactive with OMPs prepared from the serumsensitive strain as determined by ELISA. When adjusted for LOS titer present in whole serum, the immunopurified preparation accounted for two-thirds of the chemotactic potential and bactericidal activity of whole serum. Purified antibody and C (6\% AGS), when used with an unrelated serum-resistant strain, did not promote significant chemotaxis (CTX 105 \pm 41 ), nor did C alone when used with the serum-sensitive strain (CTX 128 \pm 40 ). The anti-LOS titer in the effluent of normal human serum, passed over the affinity column, was reduced by $87 \%$ compared to the parent serum, $(280 \pm 32$ vs. $2,150 \pm 210)$ but $95 \%$ of the antiOMP titer ( $795 \pm 81$ vs. $837 \pm 210$ ) was preserved (Table V). Chemotactic potential and bactericidal antibody titer in the effluent were reduced by $63 \%(213 \pm 58$ vs. $588 \pm 118)$ and $75 \%(1: 84$ vs. $1: 21$ ), respectively. Eluents recovered from control columns had no bactericidal or chemotaxis generating capability and effluents from the columns maintained 85 and $90 \%$ of these functions, respectively.

The mean IgG titer in NHS directed against serum-resistant LOSs was $10,085 \pm 2290$ compared with 4,671 \pm 696 (Table IV) against serum-sensitive LOSs, $(P<0.05)$. As indicated above, IgG fractions derived from NHS failed to promote significant chemotaxis of either serum-sensitive or resistant strains when

Table III. Antibody Titers in Convalescent Sera; Patient with Disseminated Infection (71-H)

\begin{tabular}{llllll}
\hline \multicolumn{3}{l}{ Reciprocal titer } & & & \\
\cline { 2 - 5 } $\begin{array}{l}\text { Day after } \\
\text { onset of } \\
\text { symptoms }\end{array}$ & IgM & LOS & OMPs & LOS & OMPs \\
\cline { 2 - 5 } \cline { 5 - 6 } 19 & $5,278 \pm 362$ & $3,319 \pm 407$ & $66,383 \pm 1207$ & $53,384 \pm 1112$ \\
$23^{*}$ & $3,823 \pm 210$ & $2,490 \pm 192$ & $41,180 \pm 1055$ & $42,327 \pm 2160$ \\
37 & $3,183 \pm 501$ & $2,460 \pm 57$ & $31,829 \pm 107$ & $24,863 \pm 620$ \\
61 & $2,697 \pm 611$ & $1,969 \pm 62$ & $29,724 \pm 992$ & $14,754 \pm 702$ \\
\hline
\end{tabular}

* Bactericidal and chemotaxis studies in this report were performed with serum from day 23. Bactericidal titers at each day have been reported earlier (14). 
Table IV. Antibody Titers (Reciprocal mean \pm SE) in Serum Fractions against Each of the Two Groups of $N$. gonorrhoeae Strains and a Single DGI Strain (71-H), and Corresponding Bactericidal Activity and CTX Generated by Each Fraction

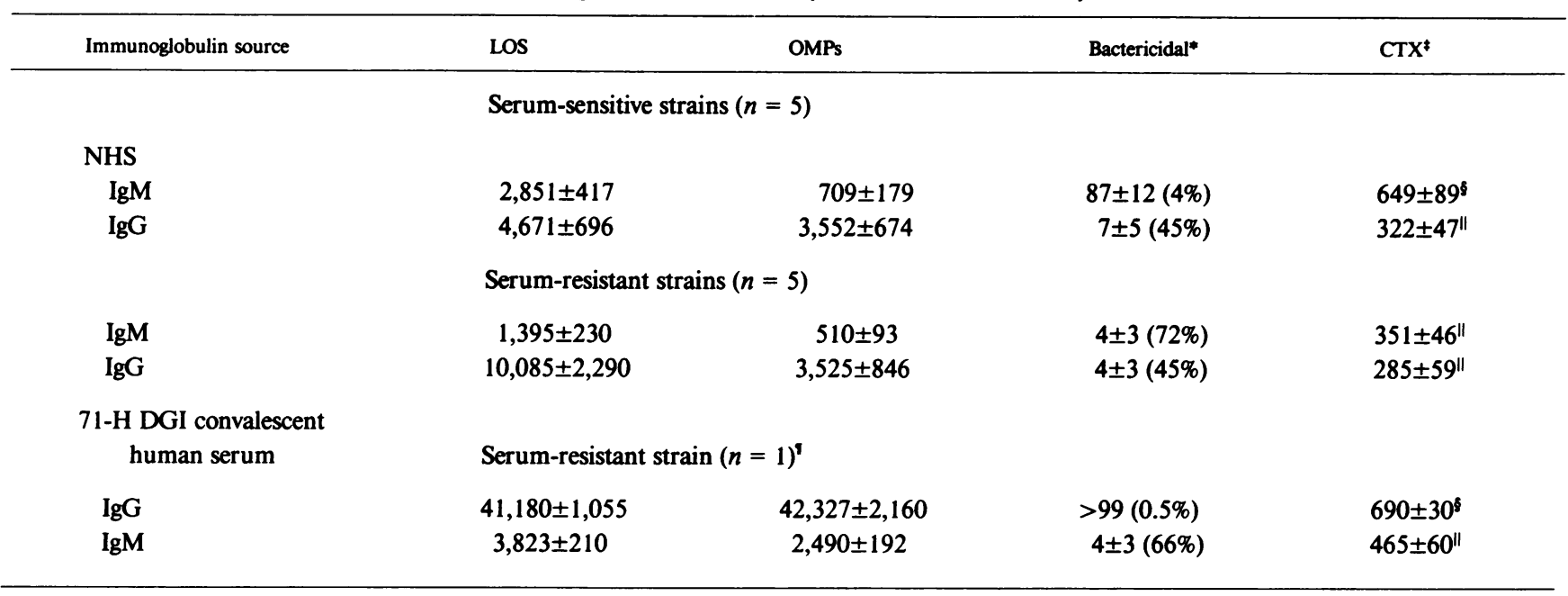

\footnotetext{
* Bactericidal activity is expressed as the mean \pm range of the $\%$ kill for each group of strains. Nos. in parentheses indicate the Ig concentration in the reaction mixtures expressed as percent in whole serum. ${ }^{\ddagger}$ Chemotaxis (expressed as $\mathrm{cpm} \pm \mathrm{SE}$ of ${ }^{51} \mathrm{Cr}$ ) generated in preincubation mixtures that contained Ig concentrations equivalent to $3 \%$ whole serum (see text). Controls that included 6\% AGS supplemented with immunoglobulins, but without gonoccci, were each $<200 \mathrm{cpm}$, and were subtracted from experimental values to provide the indicated $\mathrm{cpm}$. ${ }^{8} P<0.05$, CTX in test sample vs. 6\% AGS plus gonococci, but no Igs. " $P=$ NS, CTX in test sample vs. 6\% AGS plus gonococci, but no Igs. 'Strain 71-H.
}

reactions with each of these two groups of strains were examined as a whole (Fig. 1 and Table IV). However, chemotaxis generated by individual serum-resistant strains preincubated with IgG prepared from NHS showed a linear correlation with IgG titers against individual whole LOSs ( $r=0.87$, Table II). Two strains, when opsonized with IgG having high titered activity against the respective LOSs $(18,621 \pm 1,061$ and $10,471 \pm 898)$ promoted chemotaxis greater than 2 SE above the AGS control (Table II). IgG titers against OMPs showed no correlation with IgG mediated chemotaxis of serum-sensitive or resistant strains. In addition, no significant difference between the mean titers against serum-sensitive vs. resistant OMPs was observed (Table IV).IgG fractions from 71-H DGI convalescent serum with a homologous anti-LOS titer of $41,180 \pm 1,055$ and bactericidal activity specific for LOS (14), supported significant chemotaxis of the infecting resistant strain when used at a concentration $(0.5 \%$ convalescent serum vs. $3 \%$ normal serum) that contained $97 \%$ of the (calculated) IgG-LOS titer in pooled normal human serum directed against this serum-resistant LOS. As with IgM, the inability of IgG in normal serum to promote significant chemotaxis by most isolates of resistant gonococci tested may not have been due solely to an insufficient titer of IgG antibodies measured by ELISA to whole LOS extracts of these strains.

LOS determinants recognized by anti-LOS antibodies. We examined the specificity of IgM and IgG antibodies directed against LOS antigens in Western blotting experiments in order to explore the possibility that isotype specific LOS antibodies (normal and convalescent) may have recognized separate LOS determinants not distinguished by an ELISA that employed whole LOS extracts as target antigens. These experiments were prompted by our observations (see above) that levels of chemotactic activity were not dictated solely by the height of LOS

Table V. Antibody Titers (Reciprocal)* in IgM Fractions Derived from a Single NHS and the Corresponding CTX Generated by Incubating Each of the Fractions with the Serum-sensitive N. gonorrhoeae Strain (24-1), the LOS of Which was Used to Prepare the Immunopurified IgM

\begin{tabular}{lllll}
\hline & LOS & OMPs & Bactericidal $^{*}$ & CTX $^{\mathbf{*}}$ \\
\hline Serum fractions & & & & $588 \pm 118$ \\
NHS & $2,150 \pm 210$ & $837 \pm 210$ & 84 & $603 \pm 207$ \\
IgM fraction" & $2,230 \pm 150$ & $714 \pm 172$ & 84 & $609 \pm 90^{\ddagger \pm}$ \\
IgM immunopurified (eluent) & $3,345 \pm 601$ & $<30^{* *}$ & 84 & $213 \pm 58$ \\
NHS-immunodepleted (effluent) & $280 \pm 32$ & $795 \pm 81$ & 21 & \\
\hline
\end{tabular}

\footnotetext{
* Geometric mean titers (IgM-ELISA) of three determinations \pm SE. ${ }^{\ddagger}$ Bactericidal assay described in the text. ${ }^{8}$ Chemotaxis (expressed as counts per minute of ${ }^{51} \mathrm{Cr}$ ) generated in preincubation reaction mixtures containing $3 \%$ of the designated serum fraction (see text). "IgM fraction (prepared by gel filtration over Sephacryl G-300). ' 'IgM immunopurified against serum-sensitive $N$. gonorrhoeae LOS-hydrazide was concentrated until the bactericidal titer was equivalent to that in NHS and IgM prepared from NHS (1:84). ** Readings at lowest dilution tested (1:30) equaled negative controls. ${ }^{\ddagger}$ Controls included CTX measurements when IgM immunopurified and complement together were used with an unrelated serum-resistant strain $(C T X=105 \pm 41)$ or when complement alone was used with the corresponding-sensitive strain $(C T X=128 \pm 40)$.
} 
antibody titers present in sera or derived immunoglobulin fractions used for opsonization. Separated components of LOSs from a serum-sensitive strain (24-1) and the resistant strain (71-H) isolated from the DGI patient whose immune serum was used in these studies were employed as target antigens. IgM antibodies in NHS recognized a broad band of a faster moving component(s) of serum-sensitive LOS (Fig. 3, band $A$ in lane 3 ) that was incompletely seen by IgG (lane 4 ). This component was not visualized on gels stained with silver nitrate (lane 2) (25) compared to slower moving LOS bands that were recognized both by IgM and IgG in NHS (lanes 3 and 4). When tested against serumresistant LOS, normal but nonchemotaxic promoting IgM antibodies recognized the same LOS determinants that were identified by convalescent immune IgM (lanes 6 and 7, respectively), the latter having more than twice the titer measured in NHS, yet still unable to promote CTX when opsonized to the infecting strain. Two separate and distinct bands $(C$ and $D$ in lanes 6 and 7) were recognized by IgM and were not clearly recognized by IgG (lanes 8 and 9). Silver stained SDS polyacrylamide gel (lane 5) failed to demonstrate the fastest moving component (band D) of serum-resistant (71-H) LOS, analogous to the situation with LOS from the serum-sensitive strain (24-1).

Antibody binding of convalescent immune IgG to serumresistant LOS was distinguished by its recognition of a faster moving component (band $B^{\prime}$ in lane 8 ) that was seen faintly by IgG antibody in NHS (lane 9) but not by IgM antibody in NHS or in convalescent serum (lanes 6 and 7). The location of band $B^{\prime}$ corresponds to a faint but separately stained band (band $B$ on lane 5) on the gel of serum-resistant LOS. In separate experiments (not shown) we have demonstrated that antigenic specificity of convalescent immune IgG for this component (band $B$ ) on serum-resistant LOS is shared, at least in part, with a monoclonal antibody, named 2-1-L8, (kindly provided by W. D. Zollinger), recently reported to recognize an LOS epitope

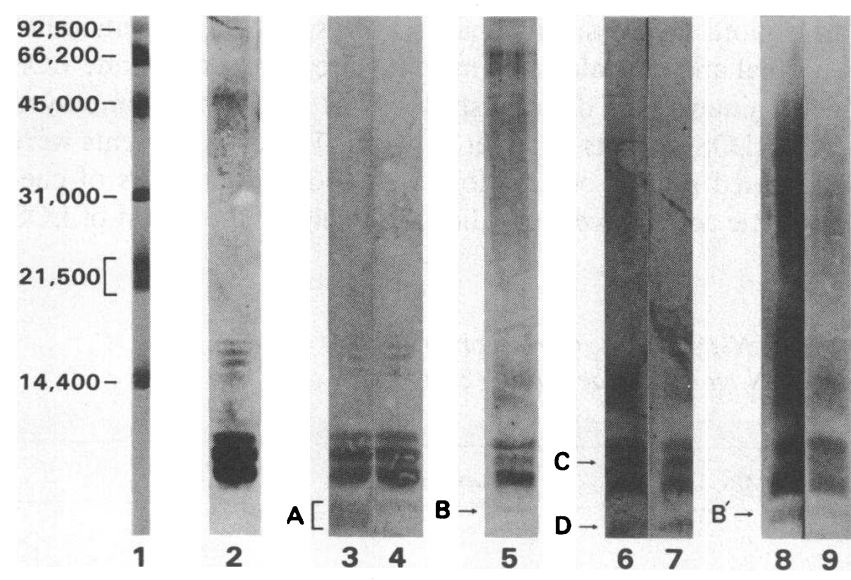

Figure 3. Analysis by Western blotting of reactivity of normal and convalescent immune (DGI) serum against serum-sensitive (24-1) and resistant (71-H) LOS antigens. Lane 1, standards; lane 2, SDS-PAGE ( $14 \%$ acrylamide) of serum-sensitive LOS (silver stained); lane 3, normal human serum IgM reacted with serum-sensitive LOS in Western blot; lane 4, normal IgG reacted with serum-sensitive LOS; lane 5, SDS-PAGE of serum-resistant LOS (silver stained); lane 6, normal IgM reacted with resistant LOS in Western blot; lane 7, convalescent immune (DGI) serum (patient 71-H) IgM reacted with resistant LOS; lane 8, immune IgG reacted with serum-resistant LOS; lane 9, normal IgG reacted with resistant LOS. present on some strains of serum-resistant but not serum-sensitive $N$. gonorrhoeae (44). 2-1-L8 antibody also manifests bactericidal activity against strains that resist killing by NHS when it is used together with human complement (44).

\section{Discussion}

Clinically varied types of gonococcal infection are caused by diverse strains of $N$. gonorrhoeae with unique inflammatory potential. The inflammatory responses elicited in human hosts by $N$. gonorrhoeae are the culmination of direct phlogistic effects caused by the organism or its constituents as well as contributing immunologic responses by the host. $N$. gonorrhoeae that resist killing by NHS cause DGI and usually produce no symptoms (e.g., inflammation) at local genital sites (6). Strains sensitive to NHS killing frequently are responsible for the most severe cases of local inflammatory disease, particularly acute salpingitis (2). Purulent discharge containing numerous PMN is the hallmark of disease in women with acute salpingitis or cervicitis and in males with symptomatic urethritis. The absence of a discharge is characteristic of males with asymptomatic urethritis.

The basis for the unique inflammatory potential exhibited by different gonococci is poorly understood. The capacity of these organisms to stimulate PMN chemotaxis in vitro has been examined as a measure of this potential. Both complement independent and complement-dependent factors have been described $(3,45-47)$. The former have been detected in filtrates of broth cultures of gonococci (46) and in gonococcal lysates (47). The magnitude of this chemotactic stimulus is small when compared with that generated by either broth filtrates of other organisms (48) or a complement dependent factor (C5a) produced during incubation of gonococci in serum $(3,45)$. Nevertheless, strains with different clinical potential may harbor intrinsically different chemotactic promoting potential that is manifested in the absence of serum and which may be important where concentrations of serum factors are low (49). In these and earlier studies (3), we have established that such a relationship also exists for complement-dependent chemotactic factors generated by gonococci isolated from patients with either symptomatic local disease or DGI. These findings have provided an experimental basis for the clinical observations described above. The development of a prompt complement dependent inflammatory response may be a critical determinant in the local confinement of infection since the absence of this response is associated with an increased likelihood that gonococcal infection will spread systemically.

Information that describes precisely how gonococci interact with the host to stimulate local immunity is incomplete. For example, most strains that cause DGI do not provoke an overt local inflammatory response $(6,7)$. All classes of immunoglobulins are present in cervical and uterine secretions and they increase during the luteal or secretory phase of the menstrual cycle $(42,43)$. Local antibodies directed against gonococcal surface antigens are present during infection (50), but their role in the initiation of inflammation or in mediating protection is incompletely defined. In addition, complement proteins are present in normal cervical mucus $(42,43)$ and low levels of intact total hemolytic complement activity (11.5\% that in serum) have been measured in normal secretions (13). While the presence of specific antibody and complement may seem advantageous, enabling the host to eradicate the bacteria via an inflammatory sequence, the consequences of the inflammation may be dele- 
terious and contribute to the morbidity associated with these infections. Nevertheless rapid and efficient complement-dependent serum-bactericidal activity and neutrophil accumulation are components of the host response to $N$. gonorrhoeae that may be important in preventing DGI. The combination of antibodies measured in female genital secretions directed against specific gonococcal antigens, such as LOS (50), and the presence of complement suggest a mode of perpetuating inflammation that may result in a PMN leukocyte response accompanied by symptomatic local disease.

Our data indicate a role for antibody in determining the pattern of neutrophil chemotaxis stimulated by serum-sensitive and resistant gonococci in a manner analogous to its role in complement dependent killing of gonococci. These two immunologic mechanisms (chemotaxis and bactericidal reactivity) have in common their facilitation by antibody and complement (1-3). Normal serum contains "natural" bactericidal antibodies of the IgM class $(2,11)$ which are also effective in promoting chemotaxis by serum-sensitive strains. In contrast, serum-resistant strains that react with IgM from normal or convalescent serum do not stimulate chemotaxis indicating that the interaction of IgM with serum-resistant gonococci does not result in the generation of the C5a necessary for chemotaxis. More imortant, ELISA testing for antibody of the IgM isotype in NHS measured against whole LOS antigens, demonstrated a direct relationship between the height of the specific anti-LOS titer and the magnitude of the chemotactic response induced by serum-sensitive but not resistant strains. In contrast, a relationship between the height of IgG antibody titers for resistant LOS antigens was correlated with the magnitude of the chemotactic response induced by serum-resistant but not sensitive strains. While the magnitude of the latter response was meager in NHS, it was prominent in convalescent (DGI) serum.

Numerous studies in addition to this one have confirmed that antibodies directed against LOS antigens of $N$. gonorrhoeae are responsible for complement-dependent bactericidal activity $(12,14,50,51)$. This activity has been ascribed to LOS antibodies of the IgM class in NHS, directed against serum-sensitive strains $(12,52-53)$ or in the case of immune DGI serum, IgG antibodies against serum resistant LOSs that kill these strains $(14,54)$. In the studies reported here, we have shown that immunopurified LOS specific IgM, lacking detectable antibody against OMP, promotes chemotactic activity by serum-sensitive gonococci and also mediates complement dependent bactericidal activity. Moreover, it accounted for nearly two-thirds of these activities in NHS. Remaining bactericidal and chemotactic activities in normal and patient convalescent sera may reside with antibodies having separate specificity. Indeed, antibodies directed against gonococcal porin (PI) present in convalescent DGI sera have been shown to contribute to bactericidal activity (55) which was otherwise primarily dictated by LOS antibodies (55). The presence of blocking antibodies directed against PIII (56), an antigenically common gonococcal membrane protein $(57,58)$, may preclude detection of bactericidal activity in sera that have immunologic specificity for mixtures of OMPs that contain both PI and PIII. This may partially explain why in the current and earlier (14) studies, bactericidal activity directed against OMPs was minimal or absent.

In separate experiments, we have shown that incubation in NHS, of the serum-sensitive LOS, used in these studies (24-1) to prepare the immunoabsorbant, results in the generation of chemotactic $\mathrm{C5a}$ in a time and concentration dependent fashion.
In contrast, serum-resistant (71-H) LOS when incubated in NHS failed to generate chemotactic activity (unpublished observations). In addition, we also found in the present studies that although the mean IgM titer in NHS for serum-resistant LOSs was lower than for serum-sensitive LOSs, two individual sera (1 normal, 1 immune convalescent) had IgM titers against a serumresistant LOS exceeding those against sensitive LOSs, but were also ineffective in promoting chemotaxis by the resistant strain. In other experiments we have found that the kinetics and amount of IgM binding to serum-sensitive and resistant strains incubated in NHS is equal over the range of serum concentrations employed in the present chemotaxis studies (unpublished observations). Thus, diminished chemotaxis by serum-resistant gonococci in NHS is not accounted for solely on the basis of a lower concentration of IgM measured by ELISA against whole LOS antigens of serum-resistant compared with sensitive organisms or by reduced IgM binding to the resistant isolates. The finding of equivalent IgM binding but different degrees of complement dependent chemotaxis generated by serum-sensitive and resistant strains suggests that complement activation may be modulated at a step prior to C5 cleavage on serum-resistant strains. In contrast to IgG from NHS, we found that IgG antibody from DGI convalescent serum directed against LOS from the homologous infecting strain supported chemotaxis of this strain. Moreover its demonstrated potential was expressed at a titer, measured by ELISA against whole LOS antigen, that in NHS was incapable of supporting chemotaxis by this strain.

We sought to explain these observations by an examination of the immunochemical specificity of IgM and IgG antibodies for components of whole LOS antigens from a serum-sensitive (24-1) and resistant (71-H) strain, separated as bands by SDSPAGE. Recently reported studies have shown that LOS epitopes may be separated by SDS-PAGE, as evidenced by distinct binding of LOS-specific murine monoclonal antibodies to separated LOS bands in Western blotting experiments $(31,44,52)$. We found that IgM antibodies from NHS which had chemotactic and bactericidal capability for the serum-sensitive strain, recognized a broad, faster migrating LOS component(s) that was incompletely recognized by normal IgG that lacked these functions. In our studies, faster migrating components may represent minor components of LOS with shorter fatty acid composition, or components with insufficient carbohydrate content to oxidize and stain with silver; nonetheless, separate evidence for LOS subspecies binding of IgM as a possible requirement for serum killing has also been provided recently by other studies demonstrating that serum-sensitive $N$. gonorrhoeae induced to serumresistance lose an LOS band that binds IgM in NHS (59).

We also observed a distinct location of binding by bactericidal and chemotactic IgG from DGI convalescent serum to a band on the LOS of the infecting serum-resistant strain. This band was not recognized by IgM in immune convalescent serum and was minimally recognized by IgG in NHS, neither of which had bactericidal or chemotactic function against this strain. The unique LOS epitope recognized by convalescent IgG antibody on LOS blots is identical in location to the faster migrating LOS epitope recognized by the monoclonal antibody, 2-1-L8 (44). Furthermore, convalescent IgG competes for binding to the antigen recognized by 2-1-L8 monoclonal antibody (unpublished observations). As indicated earlier, LOS antigens derived from serum-sensitive and serum-resistant organisms may be uniquely distinguished by this monoclonal antibody (44) which has been shown to recognize some serum-resistant (e.g., 71H), but not 
sensitive (e.g., 24-1) gonococcal LOS antigens, and to manifest bactericidal activity for strains that resist killing by NHS (44).

Thus, the characteristics and diversity in function of human antibodies both in response to specific antigenic challenge and present as natural antibody against gonococcal LOSs, may be linked to the distinct immunochemical specificity for antigenic determinants within LOS antigens. While the precise mechanism by which this occurs requires further delineation, our findings suggest that unique determinants on the same or related LOS molecules may also differ in their phlogistic potential when recognized by specific immune and natural antibodies of diverse isotypes.

\section{Acknowledgments}

We wish to thank Linda A. MacKeen, Harold E. Vayo, Connie M. McRill, for excellent technical assistance and Luzvisminda D. Recla, for assisting in the preparation of the manuscript.

This research was supported by grants AI 15633, AI 16476 and AI 20880 from The National Institutes of Health.

\section{References}

1. Schoolnik, G. K., T. M. Buchanan, and K. K. Holmes. 1976. Gonococci causing disseminated infection are resistant to the bactericidal action of normal human sera. J. Clin. Invest. 58:1163-1173.

2. Rice, P. A., W. M. McCormack, and D. L. Kasper. 1980. Natural serum bactericidal activity against Neisseria gonorrhoeae from disseminated, locally invasive, and uncomplicated disease. J. Immunol. 124: 2105-2109.

3. Densen, P., L. A. MacKeen, and R. A. Clark. 1982. Dissemination of gonococcal infection is associated with delayed stimulation of complement-dependent neutrophil chemotaxis in vitro. Infect. Immun. 38: 563-572.

4. Kaspar, R. L., and D. J. Drutz. 1977. Perihepatitis and hepatitis as complications of experimental endocarditis due to Neisseria gonorrhoeae in the rabbit. J. Infect. Dis. 136:37-41.

5. Crawford, G., J. S. Knapp, J. Hale, and K. K. Holmes. 1977. Asymptomatic gonorrhea in men: caused by gonococci with unique nutritional requirements. Science (Wash. DC). 205:1352-1353.

6. O'Brien, J. P., D. L. Goldenberg, and P. A. Rice. 1983. Disseminated gonococcal infection: A prospective analysis of 49 patients and a review of pathophysiology and immune mechanisms. Medicine. 62:395406.

7. Rice, P. A., and D. L. Goldenberg. 1981. Clinical manifestations of disseminated infection caused by Neisseria gonorrhoeae are linked to differences in bactericidal reactivity of infecting strains. Ann. Intern. Med. 95:175-178.

8. Ross, S. C., and P. Densen. 1985. Opsonophagocytosis of Neisseria gonorrhoeae: Interaction of local and disseminated isolates with complement and neutrophils. J. Infect. Dis. 151:33-41.

9. Brogadir, S. P., B. M. Schimmer, and A. R. Myers. 1979. Spectrum of gonococcal arthritis-dermatitis syndrome. Semin. Arthritis Rheumatol. 8:177-183.

10. Masi, A. T., and B. I. Eisenstein. 1981. Disseminated gonococcal infection (DGI) and gonococcal arthritis (GCA): I. Bacteriology, epidemiology, host factors, pathogen factors and pathology. Semin. Arthritis Rheumatol. 10:155-171.

11. Schoolnik, G. K., H. D. Ochs, and T. M. Buchanan. 1979. Immunoglobulin class responsible for gonococcal bactericidal activity of normal human sera. J. Immunol. 122:1771-1779.

12. Glynn, A. A., and M. E. Ward. 1970. Nature and heterogeneity of the antigens of Neisseria gonorrhoeae involved in the serum bactericidal reaction. Infect. Immun. 2:162-168.

13. Price, R. J., and B. Boettcher. 1979. The presence of complement in human cervical mucus and its possible relevance to infertility in women with complement-dependent serum-immobilizing antibodies. Fert. Steril. 32:61-66.

14. Rice, P. A., and D. L. Kasper. 1977. Characterization of gonococcal antigens responsible for induction of bactericidal antibody in disseminated infection. J. Clin. Invest. 60:1149-1158.

15. James, J. F., and J. Swanson. 1978. Studies on gonococcus infection. XIII. Occurrence of color/opacity colonial variants in clinical cultures. Infect. Immun. 19:332-340.

16. Gnehm, H. E., S. I. Pelton, S. Gulati, and P. A. Rice. 1985. Characterization of antigens from nontypable Haemophilus influenzae recognized by human bactericidal antibodies. J. Clin. Invest. 75:16451658.

17. Westphal, O., O. Luderitz, and F. Bister. 1952. Uber die Extraktion von Bacterien mit Phenol/Wasser. Z. Naturforsch. B7:148-155.

18. Dubois, M., K. A. Gilles, J. K. Hamilton, P. A. Reber, and F. Smith. 1956. Colorimetric method for determination of sugars and related substances. Anal. Chem. 28:350-356.

19. Weissbach, A., and J. Hurwitz. 1959. The formation of 2-keto3-deoxy-heptonic acid in extracts of Escherichia coli B. J. Biol. Chem. 234:705-709.

20. Osborn, M. J. 1963. Studies of the Gram negative cell wall. I. Evidence for the role of 2-keto-deoxyoctonate in the lipopolysaccharide of Salmonella typhimurium. Proc. Natl. Acad. Sci. USA. 50:499-506.

21. Lowry, O. H., N. J. Rosebrough, A. L. Farr, and R. J. Randall. 1951. Protein measurement with the Folin phenol reagent. J. Biol. Chem. 193:265-275.

22. Perry, M. B., V. Daoust, K. G. Johnson, B. B. Diena, and F. E. Ashton. 1978. Gonococcal R-Type Lipopolysaccharides. In Immunobiology of Neisseria gonorrhoeae. G. E. Brooks, E. C. Gotschlich, K. K. Holmes, W. D. Sawyer, and F. E. Young, editors. American Society for Microbiology, Washington, DC, p. 101.

23. Laemmli, U. K. 1970. Cleavage of structural proteins during the assembly of the head of bacteriophage $T_{4}$. Nature (Lond.). 227:680-685.

24. O'Farrell, P. N. 1975. High resolution two-dimensional electrophoresis of proteins. J. Biol. Chem. 250:4007-4021.

25. Tsai, C.-M., and C. E. Frasch. 1982. A sensitive silver stain for detecting lipopolysaccharides in polyacrylamide gels. Anal. Biochem. 119: 115-119.

26. Merril, C. R., D. Goldman, S. A. Sedman, and M. M. Ebert. 1981. Ultrasensitive stain for proteins in polyacrylamide gels shows regional variation in cerebrospinal fluid proteins. Science (Wash. DC). 211:1437-1438.

27. Switzer, R. C. III, C. R. Merril, and S. Shifrin. 1979. A highly sensitive silver stain for detecting proteins and peptides in polyacrylamide gels. Anal. Biochem. 98:231-237.

28. Hase, S., and E. Th. Rietschel. 1976. Isolation and analysis of the lipid A backbone: Lipid A structure of lipopolysaccharides from various bacterial groups. Eur. J. Biochem. 63:101-107.

29. Seiffer, S., P. M. Gallop, S. Michaels, and E. Meilman. 1960. Analysis of hydroxamic acids and hydrazides; Preparation and properties of dinitrophenyl derivatives of hydroxamic acids, oximes, hydrazides and hydrazones. J. Biol. Chem. 235:2613-2618.

30. Connelly, M. C., and P. Z. Allen. 1983. Chemical and immunochemical studies on lipopolysaccharides from pyocin 103-sensitive and -resistant Neisseria gonorrhoeae. Carb. Res. 120:171-186.

31. Schneider, H., T. L. Hale, W. D. Zollinger, R. C. Seid, C. A. Hammock, and J. McL. Griffiss. 1984. Heterogeneity of molecular size and antigenic expression within lipooligosaccharides of individual strains of Neisseria gonorrhoeae and Neisseria meningitidis. Infect. Immun. 45: 544-549.

32. Apicella, M. A., and N. C. Gagliardi. 1979. Antigenic heterogeneity of the non-serogroup antigen structure of Neisseria gonorrhoeae lipopolysaccharides. Infect. Immun. 26:870-874.

33. Kohn, J., and M. Wilchek. 1982. A new approach (cyano-transfer) for cyanogen bromide activation of sepharose at neutral $\mathrm{pH}$, which yields activated resins, free of interfering nitrogen derivatives. Biochem. Biophys. Res. Commun. 107:878-884.

34. Kohn, J., and M. Wilchek. 1981. Procedures for the analysis of 
cyanogen-bromide-activated Sepharose or Sephadex by quantitative determination of cyanate esters and imidocarbonates. Anal. Biochem. 115: 375-382.

35. Horigome, T., and H. Sugano. 1983. A rapid method for removal of detergents from protein solution. Anal. Biochem. 130:393-396.

36. Engvall, E., and P. Perlmann. 1972. Enzyme-linked immunosorbent assay, ELISA. J. Immunol. 109:129-135.

37. Towbin, H., T. Staehelin, and J. Gordon. 1979. Electrophoretic transfer of proteins from polyacrylamide gels to nitrocellulose sheets: procedure and some applications. Proc. Natl. Acad. Sci. USA. 76:43504354.

38. Blake, M. S., K. G. Johnson, G. J. Russell-Jones, and E. C. Gotschlich. 1984. A rapid sensitive method for detection of alkaline phosphatase conjugated anti-antibody on western blots. Anal. Biochem. 136:175-179.

39. Kasper, D. L., P. A. Rice, and W. M. McCormack. 1977. Bactericidal antibody in genital infection due to Neisseria gonorrhoeae. $J$. Infect. Dis. 135:243-251.

40. Rice, P. A., and D. L. Kasper. 1982. Characterization of serum resistance of Neisseria gonorrhoeae that disseminate. Roles of blocking antibody and gonococcal outer membrane protein. J. Clin. Invest. 70: 157-167.

41. Gallin, J. R., R. A. Clark, and H. R. Kimball. 1973. Granulocyte chemotaxis: An improved in vitro assay employing ${ }^{51} \mathrm{Cr}$-labelled granulocytes. J. Immunol. 110:233-240.

42. Schumacher, G. F. B. 1970. Biochemistry of cervical mucus. Fertil. Steril. 21:697-705.

43. Schumacher, G. F. B. 1973. Soluble proteins in cervical mucus. In The Biology of the Cervix. R. J. Blandau, and K. Moghissi, editors. University of Chicago Press, Chicago, IL. p. 201.

44. Schneider, H., J. McL. Griffiss, R. E. Mandrell, and G. A. Jarvis. 1985. Elaboration of a 3.6-kilodalton lipooligosaccharide, antibody against which is absent from human sera, is associated with serum-resistance of Neisseria gonorrhoeae. Infect. Immun. 50:672-677.

45. Watt, P. J., and R. Medlen. 1978. Generation of chemotaxins by gonococci. In Immunobiology of Neisseria gonorrhoeae. G. F. Brooks, E. C. Gotschlich, K. K. Holmes, W. D. Sawyer, and F. E. Young, editors American Society for Microbiology, Washington, DC. p. 239.

46. James, A. N., and R. P. Williams. 1978. Chemotactic factor(s) of Neisseria gonorrhoeae. Curr. Microbiol. 1:341-343.

47. Rank, E. L., and B. Holmes. 1984. Chemotaxis of human polymorphonuclear leukocytes toward Neisseria gonorrhoeae. J. Med. Microbiol. 17:45-52.

48. Berman, M. J., R. L. Guerrant, F. Murad, S. H. Richardson, D. Weaver, and G. L. Mandell. 1978. Interaction of polymorphonuclear neutrophils with Escherichia coli. J. Clin. Invest. 61:227-234.
49. Noble, R. C., M. C. Parekh, and J. V. Haley. 1985. Chemotaxis of Polymorphonuclear leukocytes from donors of different races toward Neisseria gonorrhoeae of three different auxotypes. Sex. Trans. Dis. 12: 188-192.

50. Tramont, E. C., J. Ciak, J. Boslego, D. G. McChesney, C. C. Brinton, and W. Zollinger. 1980. Antigenic specificity of antibodies in vaginal secretions during infection with Neisseria gonorrhoeae. J. Infect. Dis. 142:23-31.

51. Tramont, E. C., J. C. Sadoff, and M. S. Artenstein. 1974. Crossreactivity of Neisseria gonorrhoeae and Neisseria meningitidis and the nature of antigens involved in the bactericidal reactions. J. Infect. Dis. 130:240-247.

52. Apicella, M. A., S. A. Morse, R. E. Mandrell, R. Yamasaki, H. Schneider, and J. McL. Griffiss. 1985. Factors influencing the antigenic structure of the lipooligosaccharides of Neisseria gonorrhoeae. In The Pathogenic Neisseriae, G. K. Schoolnik, G. F. Brooks, S. Falkow, C. E. Frasch, J. S. Knapp, J. A. McCutchan, and S. A. Morse, editors. American Society for Microbiology, Washington, D.C. p. 366.

53. Apicella, M. A., M. A. J. Westerink, S. A. Morse, H. Schneider, P. A. Rice, and J. McL. Griffiss. 1986. Bactericidal antibody response of normal human serum to the lipooligosaccharide of Neisseria gonorrhoeae. J. Infect. Dis. 153:520-526.

54. Mandrell, R., J. Sugai, J. Boslego, R. Chung, P. A. Rice, and J. McL. Griffiss. 1987. Human immune response to monoclonal antibody-defined epitopes on lipooligosaccharides of Neisseria gonorrhoeae. In Proceedings of the Fifth International Pathogenic Neisseria Conference. H. C. Zanen, J. T. Poolman, and E. C. Beuvery, editors. In press.

55. Hook, E. W. III, D. A. Olsen, and T. M. Buchanan. 1984. Analysis of the antigen specificity of the human serum immunoglobulin $\mathrm{G}$ immune response to complicated gonococcal infection. Infect. Immun. 43:706709.

56. Rice, P. A., H. E. Vayo, M. R. Tam, and M. S. Blake. 1986. Immunoglobulin $\mathrm{G}$ antibodies directed against Protein III block killing of serum-resistant Neisseria gonorrhoeae by immune serum. J. Exp. Med. 164:1735-1748.

57. Judd, R. C. 1982. Surface peptide mapping of protein I and protein III of four strains of Neisseria gonorrhoeae. Infect. Immun. 37: 622-631.

58. Swanson, J., L. W. Mayer, and M. R. Tam. 1982. Antigenicity of Neisseria gonorrhoeae outer membrane proteins III detected by immunoprecipitation and western blot transfer with a monoclonal antibody. Infect. Immun. 38:668-672.

59. Tan, E. L., P. V. Patel, N. J. Parsons, P. M. V. Martin, and H. Smith. 1986. Lipopolysaccharide alteration is associated with induced resistance of Neisseria gonorrhoeae to killing by human serum. J. Gen. Microbiol. 132:1407-1413. 